\title{
Experimental and Theoretical Study on Glycolic Acid Provided Fast
}

\section{Bio/Seawater Degradable Poly(Butylene Succinate-co-Glycolate)}

Han $\mathrm{Hu},{ }^{1}$ Jiayi Li, ${ }^{2}$ Ying Tian, ${ }^{1}$ Chao Chen, ${ }^{1}$ Fenglong Li, ${ }^{1}$ Wu Bin Ying, ${ }^{1}$ Ruoyu Zhang $^{1 *}$ and Jin $\mathrm{Zhu}^{1 *}$

${ }^{1}$ Key Laboratory of Bio-based Polymeric Materials Technology and Application of Zhejiang Province, Ningbo Institute of Materials Technology and Engineering, Chinese Academy of Sciences, Ningbo 315201, People's Republic of China

2 State Key Laboratory of Microbial Metabolism, Joint International Research Laboratory of Metabolic \& Developmental Sciences, School of Life Sciences \& Biotechnology, Shanghai Jiao Tong University, Shanghai, 200240, China.

Corresponding authors:

zhangruoy@nimte.ac.cn (Ruoyu Zhang)

jzhu@nimte.ac.cn (Jin Zhu)

\section{Contents:}

Total number of pages: 9

Total number of equations: 6

Total number of Figures: 5

Total number of Tables: 2 


\section{Characterization Methods of PBSGAs.}

Intrinsic viscosity was tested by a Ubbelohde viscometer (inner diameter $0.792 \mathrm{~mm}$ ) at $25{ }^{\circ} \mathrm{C}$, with $125 \mathrm{mg}$ of sample dissolved in $25 \mathrm{~mL}$ solvent of phenol/1,1,2,2tetrachloroethane $(50 / 50, \mathrm{w} / \mathrm{w})$.

Determination of molecular weight $\left(M_{\mathrm{w}}\right)$ and distribution (DI) were carried out using a GPC (Agilent PL-GPC220) at $40{ }^{\circ} \mathrm{C}$, with $10 \mathrm{mg}$ of sample dissolved in $10 \mathrm{~mL}$ of chloroform. Chloroform was used as the mobile phase at the flowing rate of $1 \mathrm{~mL} / \mathrm{min}$. $M_{\mathrm{n}}$ and DI were determined using polystyrene standards (3070-258000 g/mol).

The chemical compositions were confirmed by ${ }^{1} \mathrm{H}$ NMR, which were recorded on a Bruker AVIII400 NMR spectrometer at $25^{\circ} \mathrm{C}$ with deuterated chloroform as solvent. The composition $\left(\mathrm{n}_{\mathrm{GA}}\right)$, the number-average sequence length of $\mathrm{GA}$ and $\mathrm{BS}$ units $\left(\mathrm{L}_{\mathrm{GA}}\right.$, $\mathrm{L}_{\mathrm{BS}}$ ) and the degree of randomness (R) were calculated from ${ }^{1} \mathrm{H}$ NMR data.

FT-IR spectra were obtained using an attenuated total reflectance-Fourier transform infrared (ATR-FTIR) (Agilent Cary 600 Series FT-IR) spectrometer. The scan range was from $500 \mathrm{~cm}^{-1}$ to $4000 \mathrm{~cm}^{-1}$ and each sample was scanned for 32 times.

The thermal transitions were recorded with DSC (Diamond DSC, PerkinElmer) under $\mathrm{N}_{2}$ flow of $40 \mathrm{~mL} \mathrm{~min}{ }^{-1}$. The sample was firstly heated from -40 to $200{ }^{\circ} \mathrm{C}$ with $10{ }^{\circ} \mathrm{C} / \mathrm{min}$ ramp, and then the temperature was kept at $200{ }^{\circ} \mathrm{C}$ for $3 \mathrm{~min}$. Then, it was subsequently cooled to $-40^{\circ} \mathrm{C}$ and hold for $3 \mathrm{~min}$. Finally, the second heating run was performed by heating the sample to $200{ }^{\circ} \mathrm{C}$ at $10{ }^{\circ} \mathrm{C} / \mathrm{min}$ too. The melting temperature $\left(\mathrm{T}_{\mathrm{m}}\right)$ and corresponding entropy $\left(\Delta \mathrm{H}_{\mathrm{m}}\right)$, glass transition temperature $\left(\mathrm{T}_{\mathrm{g}}\right)$, cold crystallization temperature $\left(\mathrm{T}_{\mathrm{cc}}\right)$ and its entropy $\left(\Delta \mathrm{H}_{\mathrm{cc}}\right)$ were obtained.

The thermal stability was determined by TGA experiment (Mettler-Toledo TGA/DSC) in dry $\mathrm{N}_{2}$ atmosphere $(50 \mathrm{~mL} / \mathrm{min})$. The samples around $5-8 \mathrm{mg}$ were heated to $800{ }^{\circ} \mathrm{C}$ with the heating rate of $20^{\circ} \mathrm{C} / \mathrm{min}$. The temperature at $5 \%$ weight loss $\left(\mathrm{T}_{\mathrm{d}, 5 \%}\right)$ and the temperature at the maximum rate $\left(\mathrm{T}_{\mathrm{d}, \max }\right)$ were summarized.

The patterns of X-ray diffraction were obtained with Bruker AXS D8 Advance, using $\mathrm{Cu}-\mathrm{K} \alpha$ radiation $(\lambda=0.1541 \mathrm{~nm})$ in the range from 5 to $60^{\circ}$ at the rate of $5^{\circ} / \mathrm{min}$. The PBSGA samples for XRD tests were stored at room temperature for 2 weeks. 
All samples for mechanical tests and barrier tests were prepared by compression molding and cold pressing, and then conditioned at room temperature for 2 weeks before testing.

Tensile properties were obtained at $25^{\circ} \mathrm{C}$ by an Instron 5567, according to ASTM D638. The crosshead speed was $20 \mathrm{~mm} / \mathrm{min}$ and the gauge length was $15 \mathrm{~mm}$. Dumbbell-shaped specimens $(1 \times 2 \mathrm{~mm}$ in neck thickness and width $)$ were prepared.

Surface hydrophobicity values of films were determined based on measurement of the water contact angle and seawater contact angle using a WCA analyzer OCA20 (Data physics Ltd., Germany) at normal room temperature and pressure. Five drops were observed on different areas for each film and contact angles were reported as the average value \pm standard deviation.

The $\mathrm{CO}_{2}$ and $\mathrm{O}_{2}$ barrier properties of PBSGAs were performed at $23{ }^{\circ} \mathrm{C}$ by using Labthink VAC-V2. The films with surface area of $38.5 \mathrm{~cm}^{2}$ were prepared by meltpress. The water barrier properties of samples $\left(33 \mathrm{~cm}^{2}\right)$ were studied at $38{ }^{\circ} \mathrm{C}$ and $90 \%$ RH by Labthink W3-060.

Enzymatic degradation experiments of PBSGAs films (0.3-0.35 $\mathrm{mm}$ in thickness) were carried out in Phosphate Buffered Saline ( $\mathrm{pH}$ 7.4) at $37{ }^{\circ} \mathrm{C}$, catalyzed by 0.1 $\mathrm{mg} / \mathrm{mL}$ of Candida antarctica lipase B (CALB) enzymes. Meanwhile, a controlled experiment was run parallel with them in distilled water. In the marine degradation tests, the PBSGA films were added into artificial seawater at $10-15^{\circ} \mathrm{C}$. After $7 \mathrm{~d}$ intervals, the films were washed with distilled water, and dried under vacuum at $40{ }^{\circ} \mathrm{C}$ to constant weight. The media was replaced every 7 days to ensure a stable degradation. The degradation level was evaluated by the weight percentage of residue, according to the following equation (S1):

$$
\text { Residue Weight }(\%)=\frac{\mathrm{w}_{t}}{\mathrm{w}_{0}} \times 100 \%
$$

Where $W_{0}$ is the original weight, and $W_{\mathrm{t}}$ is the weight after degradation.

Storage stability tests of PBSGAs films were performed at room temperature with $70 \%$ RH. After every month of storage, recorded the intrinsic viscosity of copolyesters.

\section{Theoretical Calculation Method}


In order to obtain all stable configurations on the potential energy surface of the 3-1 complex and 5-1 complex, 100 initial complexes were randomly generated by the genmer in the cluster configuration search program Molclus ${ }^{1}$ without relying on empirical judgments. The geometry optimization and frequency analysis of these structures were performed using the extended tight-binding semiempirical program package (xTB); the geometries and energies were obtained with GFN2-xTB..$^{2-3}$ These initial geometries in a rough level conformation with relatively low energy and different geometry were screened out the isostat tool in Molclus to sort count the energy of the complexes and remove the repetitive structures with similar energy and high geometric similarity. The parameter settings used the default values in the program, i.e., the energy threshold for distinguishing clusters, $0.5 \mathrm{kcal} / \mathrm{mol}$, and the geometric threshold for distinguishing clusters, $0.25 \AA$ A. Finally, the optimized isomers were sorted in energy, and the 10 lowest-lying ones were treated with Gaussian 16 software to obtain the precise geometries at a level of B3LYP/6-31G(d,p) ${ }^{4}$ with Grimme D3 dispersion. ${ }^{5}$ The prediction of reactive sites between nucleophile and electrophile was studied by the frontier molecular orbital (FMO) analysis and with hirshfeld charges ${ }^{6}$ and with Fukui functions (FF). The Fukui function was used to predict reactive sites at the B3LYPD3/def-TZVP level of theory. Fukui function is defined as ${ }^{7-8}$ :

$=\left[\frac{\partial \rho(r)}{\partial N}\right]_{v}$

where $\mathrm{N}$ is number of electrons in present system, the constant term in the partial derivative is external potential. For a given molecular system, Fukui function was calculated using electron density of three states:

$$
\begin{aligned}
& f^{+}(r)=\rho_{N+1}(r)-\rho_{N}(r) \approx \rho^{\text {LUMO }}(r) \\
& f^{-}(r)=\rho_{N}(r)-\rho_{N-1}(r) \approx \rho^{\text {HOMO }}(r) \\
& f^{0}(r)=\frac{f^{+}(r)+f^{-}(r)}{2}=\frac{\rho_{N+1}(r)-\rho_{N-1}(r)}{2} \\
& \approx \frac{\rho^{\text {HOMO }}(r)+\rho^{\text {LUMO }}(r)}{2}
\end{aligned}
$$


where $\mathrm{N}$ is the number of electrons in the current molecular system. The $N-1$ and $N+1$ states share the same molecular geometry as the $N$ state. For nucleophiles, $f^{-}$ is the reactivity descriptor, while for electrophiles, $f^{+}$is the descriptor. $f^{0}$ is reactivity descriptor for radical attack. Atoms with larger Fukui function tend to have higher reactivities. Molecular polarity index (MPI) was also calculated, for which is expressed as:

$$
\text { MPI }=(1 / A) \iint_{S}|V(r)| d S
$$

where A and $V(r)$ refer to the area of vdW surface and value of ESP at a point $r$ in space, respectively. The integration is performed over the whole molecular vdW surface (S). MPI is a quite reliable index of measuring molecular polarity, the larger the index, the higher the polarity. ${ }^{9}$ Isosurface maps were produced using VMD 1.9.3. ${ }^{10}$ program based on outputs from the Multiwfn calculations. ${ }^{11}$

A further frequency calculation at the same level of theory were performed to confirm each stationary point to be either an intermediate or a transition structure after structural optimizations at a level of B3LYP/6-31G (d,p) with Grimme D3 dispersion. In addition, the intrinsic reaction coordinates (IRC) calculations ${ }^{12}$ and relaxed potential surface scans were carried out to identify transition states and immediate reactants and products to study the mechanism of hydrolysis. The electronic energies were updated with the single point calculation of the larger basis sets, i.e., B3LYP-D3/6-311+G(d,p). 


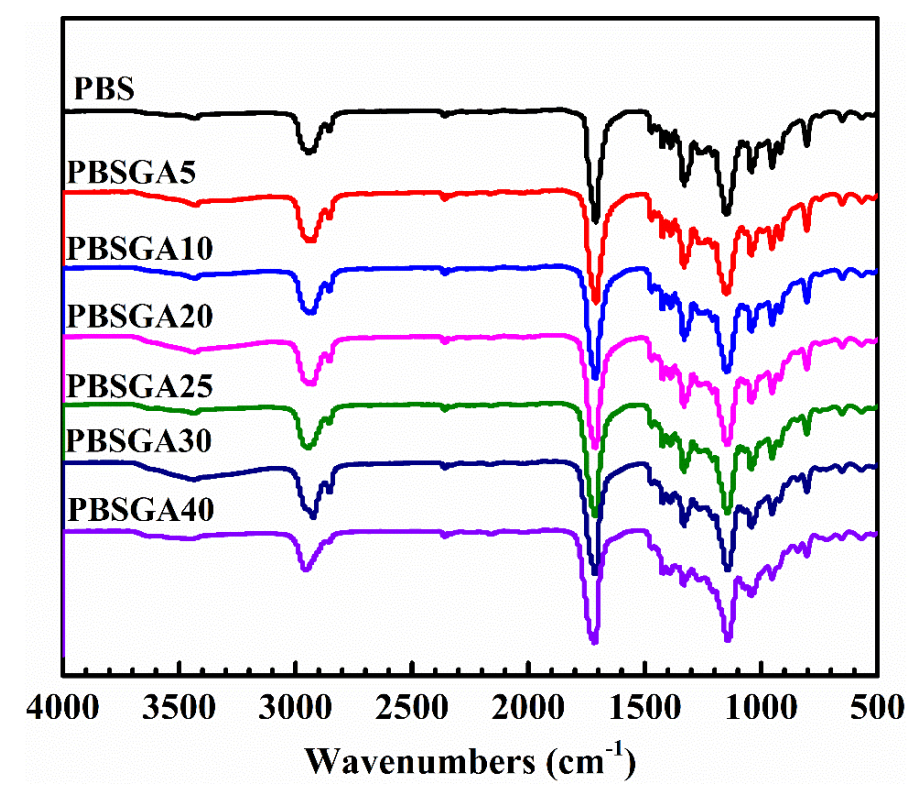

Figure S1. ATR-FTIR spectra of PBFGAs.
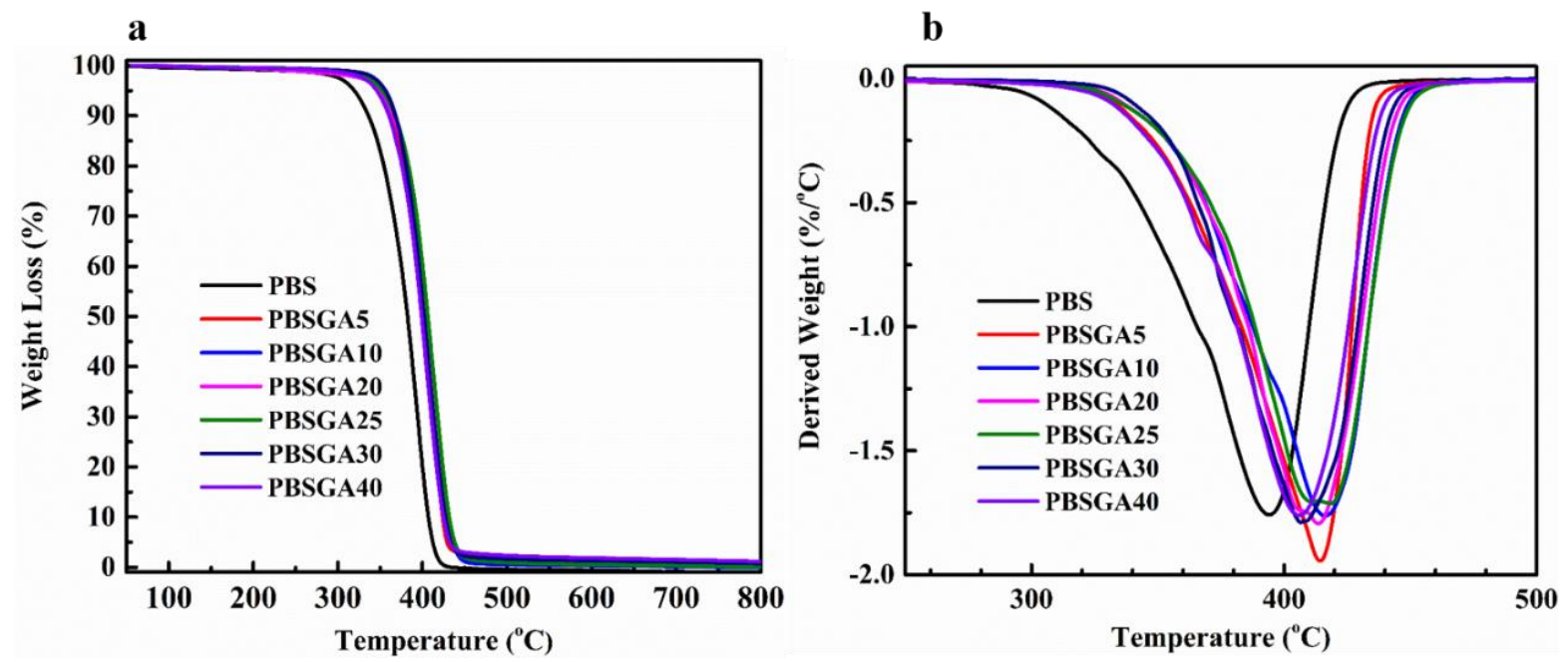

Figure S2. (a) TGA and (b) DTG curves of PBSGAs. 


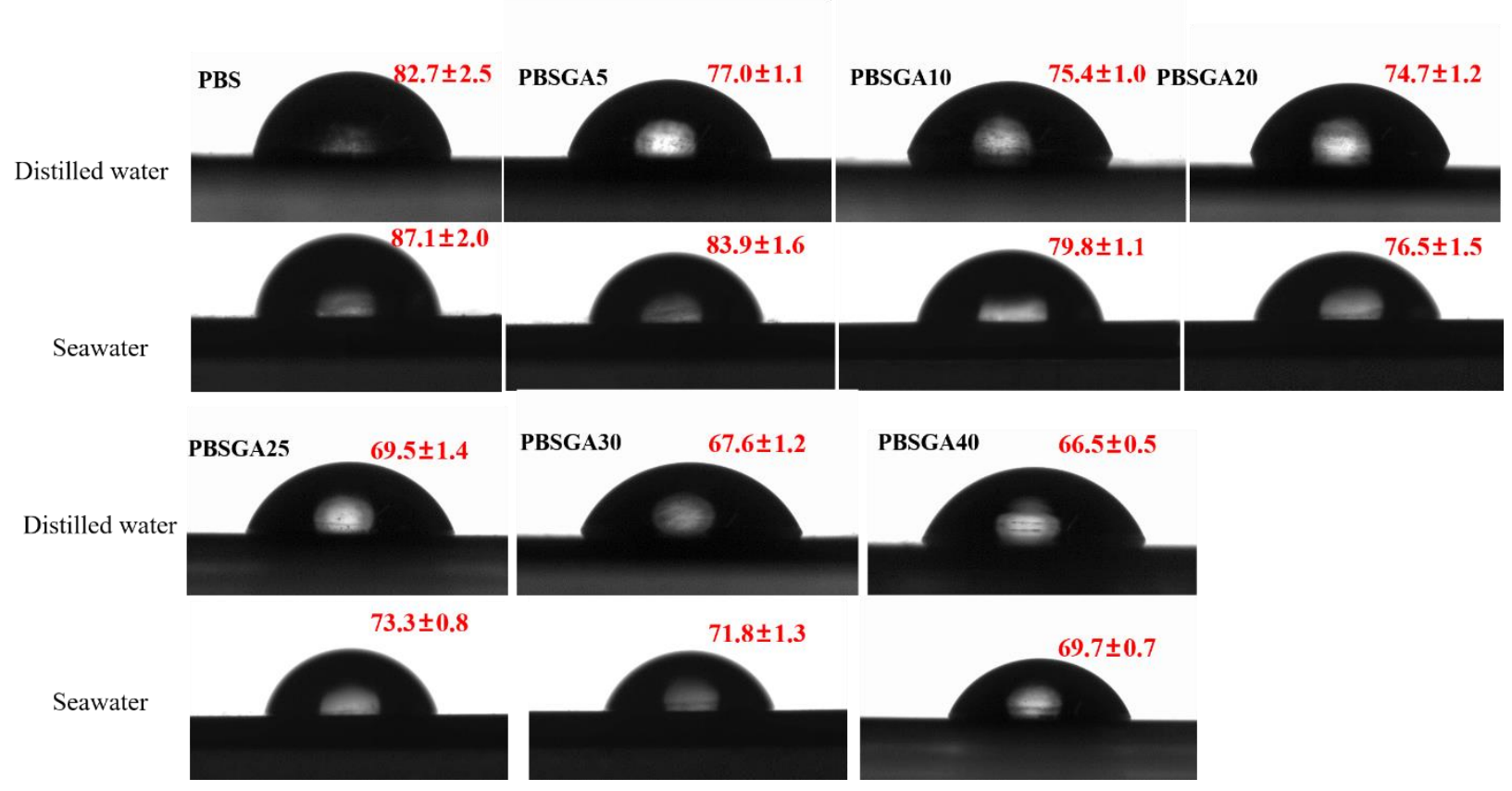

Figure S3. Distilled water and seawater contact angle images of PBSGAs.

Table S1. Molecular weight changes of PBSGAs after 49 days of seawater degradation.

\begin{tabular}{|c|c|c|c|c|}
\hline \multirow[t]{2}{*}{ Sample } & \multicolumn{2}{|c|}{ Before degradation } & \multicolumn{2}{|c|}{$\begin{array}{c}\text { After } 49 \text { days of seawater } \\
\text { degradation }\end{array}$} \\
\hline & $\mathrm{M}_{\mathrm{w}}(\mathrm{g} / \mathrm{mol})$ & DI & $\mathrm{M}_{\mathrm{w}}(\mathrm{g} / \mathrm{mol})$ & DI \\
\hline PBS & $1.18^{*} * 10^{5}$ & 2.1 & $9.88 * 10^{4}$ & 2.2 \\
\hline PBSGA5 & $1.35^{*} 10^{5}$ & 1.9 & $9.27 * 10^{4}$ & 2.3 \\
\hline PBSGA10 & $6.24 * 10^{4}$ & 2.0 & $4.60 * 10^{4}$ & 2.3 \\
\hline PBSGA20 & $8.98 * 10^{4}$ & 2.2 & $3.26 * 10^{4}$ & 2.5 \\
\hline PBSGA25 & $7.09 * 10^{4}$ & 2.3 & $2.08 * 10^{4}$ & 2.6 \\
\hline PBSGA30 & $7.73 * 10^{4}$ & 2.2 & $1.35 * 10^{4}$ & 2.5 \\
\hline PBSGA40 & $6.71 * 10^{4}$ & 2.1 & $4.06^{*} 10^{3}$ & 2.7 \\
\hline
\end{tabular}




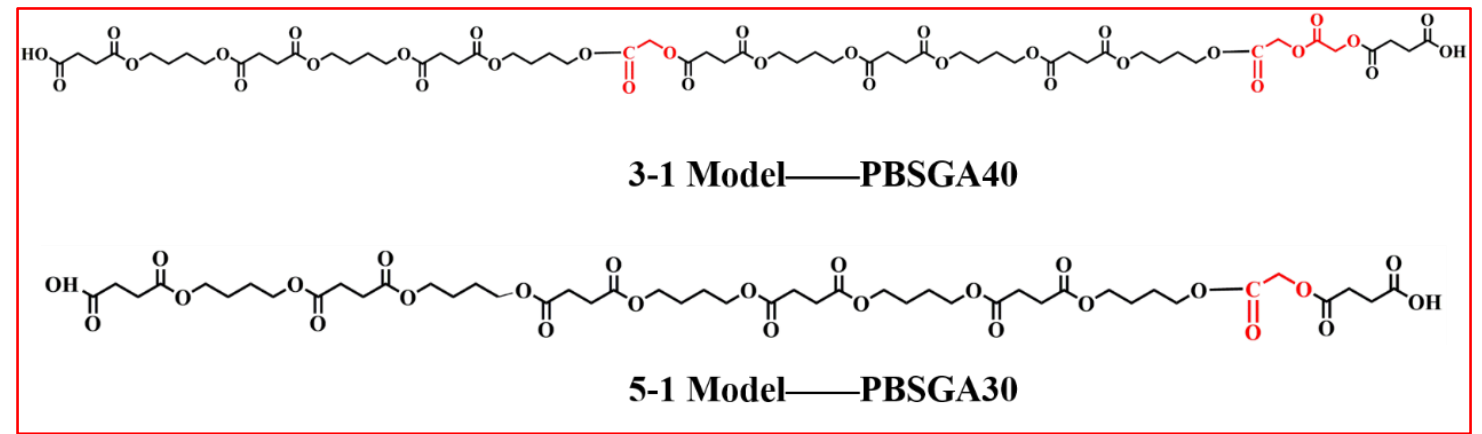

Figure S4. 3-1 and 5-1 segment model for Fukui function (FF) analysis. The 3-1 model was close to the segment of PBSGA40 and the 5-1 model was that of PBSGA30, as calculated from the ${ }^{1} \mathrm{H}-\mathrm{NMR}$ data.

A

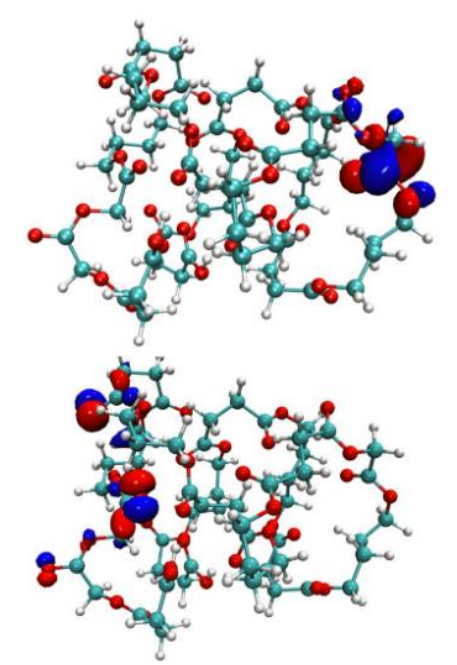

B

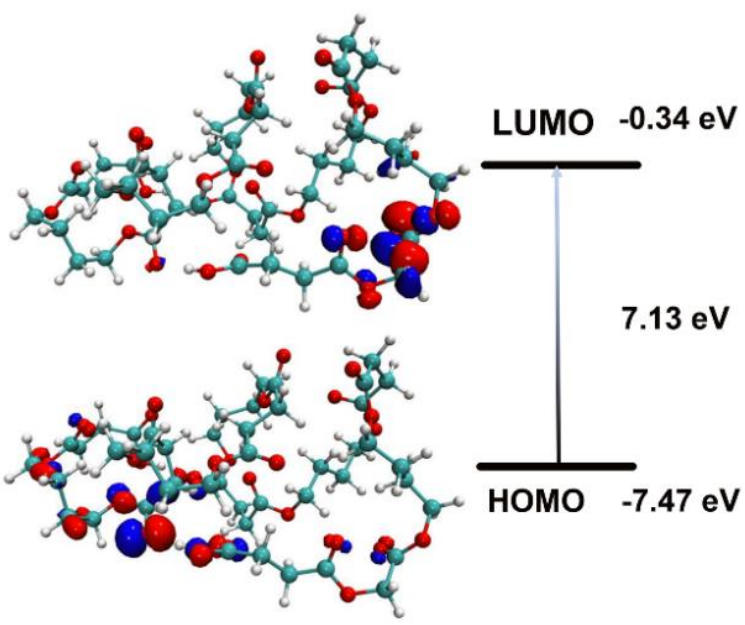

Figure S5. Prediction of the active sites by frontier molecular orbital (FMO) theory. Isosurface of the highest occupied molecular orbital (HOMO) and the lowest unoccupied molecular orbital (LUMO) of 3-1 model (A) and 5-1 model (B), respectively. Red and blue isosurfaces represent positive and negative parts, respectively.

Table S2: Comparison of the values of hirshfeld charges and Fukui functions for C1 atom of GA units in the 3-1 model and 5-1 model. The $f^{+}$can be expressed as the difference of atomic charges in two states. The atom owing larger values of $f^{+}$became the favorable sites for nucleophilic attack. 


\begin{tabular}{lllll}
\hline Atom & $\mathbf{q}(\mathbf{N})$ & $\mathbf{q}(\mathbf{N}+\mathbf{1})$ & $\mathbf{q}(\mathbf{N}-1)$ & $\boldsymbol{f}^{+}$ \\
\hline $\mathrm{C} 1(\mathbf{3 - 1})$ & 0.2161 & 0.1457 & 0.2179 & 0.0704 \\
\cline { 2 - 5 } $\mathrm{C} 1(\mathbf{5 - 1})$ & 0.2072 & 0.1610 & 0.2089 & 0.0463 \\
\hline
\end{tabular}

\section{Reference}

1. Lu, t., molclus program, Version 1.9.6, http://www.keinsci.com/research/ molclus.html (accessed July 21th, 2020).

2. Grimme, S.; Bannwarth, C.; Shushkov, P., A Robust and Accurate Tight-Binding Quantum Chemical Method for Structures, Vibrational Frequencies, and Noncovalent Interactions of Large Molecular Systems Parametrized for All spd-Block Elements (Z=1-86). J. Chem. Theory Comput. 2017, 13, 1989-2009, DOI 10.1021/acs.jctc.7b00118.

3. Bannwarth, C.; Ehlert, S.; Grimme, S., GFN2-xTB-An Accurate and Broadly Parametrized Self-Consistent Tight-Binding Quantum Chemical Method with Multipole Electrostatics and Density-Dependent Dispersion Contributions. J. Chem. Theory Comput. 2019, 15, 1652-1671, DOI 10.1021/acs.jctc.8b01176.

4. Frisch, M. J.; Trucks, G. W.; Schlegel, H. B.; Scuseria, G. E.; Robb, M. A.; Cheeseman, J. R. Gaussian 16, Revision A.03, Gaussian, Inc., Wallingford, CT, 2016.

5. Grimme, S.; Antony, J.; Ehrlich, S.; Krieg, H., A consistent and accurate ab initio parametrization of density functional dispersion correction (DFT-D) for the 94 elements H-Pu. J. Chem. Phys. 2010, 132, DOI 10.1063/1.3382344.

6. Tsuneda; Takao, Chemical reaction analyses based on orbitals and orbital energies. Int. J. Quantum Chem. 2015, 115, 270-282, DOI 10.1002/qua.24805.

7. Parr, R. G.; Yang, W. T., DENSITY FUNCTIONAL-APPROACH TO THE FRONTIER-ELECTRON THEORY OF CHEMICAL-REACTIVITY. J. Am. Chem. Soc. 1984, 106, 4049-4050, DOI 10.1021/ja00326a036.

8. Greenwood, H. H., A Molecular Orbital Theory of Reactivity in Aromatic Hydrocarbons. J. Chem. Phys. 1952, 20, 1653-1653, DOI 10.1063/1.1700243.

9. Liu, Z.; Lu, T.; Chen, Q., Intermolecular interaction characteristics of the allcarboatomic ring, cyclo[18]carbon: Focusing on molecular adsorption and stacking. Carbon 2020, 171, 514-523, DOI 10.1016/j.carbon.2020.09.048.

10. Humphrey, W.; Dalke, A.; Schulten, K., VMD: Visual molecular dynamics. J Mol Graph 1996, 14, 33-38, DOI 10.1016/0263-7855(96)00018-5.

11. Lu, T.; Chen, F., Multiwfn: A multifunctional wavefunction analyzer. J. Comput. Chem. 2012, 33, 580-592, DOI 10.1002/jcc.22885.

12. Maeda, S.; Harabuchi, Y.; Ono, Y.; Taketsugu, T.; Morokuma, K., Intrinsic Reaction Coordinate: Calculation, Bifurcation, and Automated Search. Int. J. Quantum Chem. 2015, 115, 258-269, DOI 10.1002/qua.24757. 